\title{
LA INGENIERÍA Y LA PROPIEDAD INDUSTRIAL EN EL ÁMBITO UNIVERSITARIO: MARCO LEGAL Y ALGUNAS DUDAS HABITUALES
}

\author{
Francisca Ramón Fernández*
}

\section{INTRODUCCIÓN}

Cuando se hace referencia a la patente en el seno de una Universidad surgen algunas dudas que no siempre tienen una respuesta unívoca. El estudiante que busca actualmente obtener un título de grado con el que, en un futuro, podrá trabajar dentro de un instituto, grupo de investigación o departamento que desarrolle una patente, ignora los conceptos elementales de la propiedad industrial. El diseño de los planes de estudios de los actuales grados no siempre ha sido favorable a introducir conceptos jurídicos en las ingenierías, obviándolos, y produciendo en el estudiante la carencia de unos conocimientos que le vendrían muy bien en su actividad futura.

\footnotetext{
* Doctora en Derecho, profesora titular de Derecho Civil, Universitat Politècnica de València, Valencia, España. Correo-e: frarafer@urb.upv.es Fecha de recepción: 14 de agosto de 2015. Fecha de aceptación: 15 de septiembre de 2015. El presente trabajo, cuya autora única es Francisca Ramón Fernández, es inédito, no está publicado, ni se ha remitido para su evaluación a ninguna otra revista, y se enmarca dentro de la actividad investigadora que desarrolla la autora como investigadora de los siguientes proyectos: MINECO (DER201237844), siendo el Investigador Principal el Dr. D. Lorenzo Cotino Hueso, Catedrático acreditado de Derecho Constitucional, Universitat de València-Estudi General, mineco (DER2013-4256R), siendo los Investigadores Principales la Dra. D.a Luz María Martínez Velencoso, profesora titular de Derecho Civil, Universitat de València-Estudi General, y el Dr. D. Javier Plaza Penadés, catedrático de Derecho Civil, Universitat de València-Estudi General, y el Proyecto "Derecho civil valenciano y europeo" del Programa Prometeo para Grupos de Investigación de Excelencia de la Conselleria de Educación, Cultura y Deporte, gVPrometeori2015-014, y del Microclúster "Estudios de Derecho y empresa sobre tics (Law and business studies on ICT)", dentro del vLC/Campus, Campus de Excelencia Internacional (International Campus of Excellence), coordinado por el Dr. D. Javier Plaza Penadés, catedrático de Derecho Civil, Universitat de València-Estudi General. Para citar el artículo: Ramón Fernández, F. La ingeniería y la propiedad industrial en el ámbito universitario: marco legal y algunas dudas habituales. Revista La Propiedad Inmaterial n. ${ }^{\circ}$ 20, Universidad Externado de Colombia, julio-diciembre 2015, pp. 39-56. DOI: http:// dx.doi.org/10.18601/16571959.n20.03
} 
A la pregunta: ¿es necesario explicar la propiedad industrial en los grados de la Universitat Politècnica de València (en adelante Upv)?, la respuesta es sí, por supuesto. El estudiante y el personal que investigue en la rama de Ciencias: en los grados en Biotecnología, en Ciencia y Tecnología de Alimentos, y en Ciencias Ambientales, así como los de la rama de Ingeniería y Arquitectura, Ciencia y Tecnología para la Salud con el grado en Ingeniería Biomédica, y la rama de Ingeniería y Arquitectura, Industrial y Aeronáutica, con los grados en Ingeniería Aeroespacial; en Ingeniería en Diseño Industrial y Desarrollo de Productos; en Ingeniería Electrónica Industrial y Automática; en Ingeniería Eléctrica; en Ingeniería de la Energía; en Ingeniería Mecánica; en Ingeniería de Organización Industrial; en Ingeniería Química; en Ingeniería en Tecnologías Industriales, entre otras1, y sin ánimo exhaustivo, necesita conocer cómo funciona la propiedad industrial dentro de la Universidad, y también fuera de ella, en la empresa privada2. Esto le va a dar seguridad y, sobre todo, le va a permitir resolver las preguntas: ¿si invento algo en la Universidad o en la empresa, no soy el inventor?, ¿la inventora es la Universidad o la empresa?, ¿si invento algo y no lo digo, pasa algo? A estas dudas y cuestiones vamos a intentar responder siguiendo la normativa específica de la upv y el marco legislativo general.

\section{LA INVENCIÓN DENTRO DE LA UNIVERSIDAD}

La protección de la invención mediante patente en el seno de una Universidad plantea distintos aspectos que se relacionan entre la economía, por la explotación de la patente, y la titularidad de la misma, al inventor, como sujeto, y su reconocimiento del derecho moral como inventor. La titularidad de los resultados obtenidos por el personal de la Universidad es preciso deslindarla mediante de la normativa propia de las Universidades. La invención dentro de la Universidad ${ }^{3}$ dista de la que se pueda realizar en el ámbito de una empresa, además de estar contemplado en los acuerdos de colaboración con las mismas. Un ejemplo de ello son las colaboraciones con empresas a través de la creación de spin off. En el caso de la Universitat Politècnica de València, se regula a través de la normativa sobre creación de empresas a partir de la actividad de investigación universitaria,

1. Cfr. todos los grados en: http://www.upv.es/estudios/grado/index-es.html Consultada el 24 de febrero de 2015.

2. Canós Darós, L., Ramón Fernández, F. y Mauri Castelló, J. J. "Aspectos jurídicos y económicos de la propiedad industrial de la empresa", Novática, n. ${ }^{\circ} 193$, mayojunio 2008, pp. 56-58.

3. La literatura científica en la materia es amplia; sin ánimo exhaustivo podemos citar algunas aportaciones sobre la materia: Caballero Fernández, G.; Quintás Corredoira, $M^{a}$. de los A., Arévalo Tomé, R. y Piñeiro García, Ma. P. "La protección de las invenciones mediante patentes en las universidades europeas, japonesas y estadounidenses", Cuadernos de Gestión, vol. 12 (1), 2012, pp. 15-38. 
y que fue aprobada por Consejo de Gobierno, en su sesión de 15 de mayo de $2008^{[4]}$. Hay que atender por ello a la normativa específica y resolver las dudas que se pueden generar de la lectura de la misma.

\section{La Normativa Aplicable a LA PRopiedad industrial EN EL Ámbito UNIVERSITARIO}

En cuanto a la normativa general aplicable a las patentes e invenciones que se realicen por parte de profesores en la Universidad, hay que tener en cuenta lo indicado en las siguientes normas.

1.1. Artículo 20 de la Ley 11/1986, de 20 de marzo, de Patentes':

1. Las normas del presente Título serán aplicables a los funcionarios, empleados y trabajadores del Estado, Comunidades Autónomas, Provincias, Municipios y demás Entes Públicos, sin perjuicio de lo previsto en los párrafos siguientes.

2. Corresponde a la Universidad la titularidad de las invenciones realizadas por el profesor como consecuencia de su función de investigación en la Universidad y que pertenezcan al ámbito de sus funciones docente e investigadora, sin perjuicio de lo establecido en el artículo 11 de la Ley Orgánica de Reforma Universitaria.

3. Toda invención, a la que se refiere el punto 2, debe ser notificada inmediatamente a la Universidad por el profesor autor de la misma.

4. El profesor tendrá, en todo caso, derecho a participar en los beneficios que obtenga la Universidad de la explotación o de la cesión de sus derechos sobre las invenciones mencionadas en el punto 2. Corresponderá a los Estatutos de la Universidad determinar las modalidades y cuantía de esta participación.

5. La Universidad podrá ceder la titularidad de las invenciones mencionadas en el punto 2 al profesor, autor de las mismas, pudiendo reservarse en este caso una licencia no exclusiva, intransferible y gratuita de explotación.

4. Disponible en: http://www.ctt.upv.es/docs/NormativaAprobadaConsejoGobierno_05_2008.pdf Consultado el 29 de septiembre de 2015.

5. вое n. ${ }^{\circ} 73$, de 26 de marzo de 1986. Este precepto estará vigente hasta el 1 de abril de 2017, como indica la disposición final novena, en que entrará en vigor la Ley 24/2015, de 24 de julio de Patentes (BOE n. ${ }^{\circ} 177$, de 25 de julio de 2015), y a la que haremos referencia en un momento posterior del trabajo. 
6. Cuando el profesor obtenga beneficios de la explotación de una invención mencionada en el punto 5, la Universidad tendrá derecho a una participación en los mismos determinada por los Estatutos de la Universidad.

7. Cuando el profesor realice una invención como consecuencia de un contrato con un Ente privado o público, el contrato deberá especificar a cuál de las partes contratantes corresponderá la titularidad de la misma.

8. El régimen establecido en los párrafos 2 a 7 de este artículo podrá aplicarse a las invenciones del personal investigador de Entes públicos de investigación.

9. Las modalidades y cuantía de la participación del personal investigador de Entes públicos de investigación en los beneficios que se obtengan en la explotación o cesión de sus derechos sobre las invenciones mencionadas en el punto 8 de este artículo, serán establecidas por el Gobierno, atendiendo a las características concretas de cada Ente de investigación.

La Ley 24/2015, de 24 de julio, de Patentes, regula el punto en los artículos 20 y 21. Por lo que interesa a nuestra investigación, el artículo 21 indica lo siguiente, respecto a las invenciones realizadas por el personal investigador de las Universidades Públicas y de los Entes Públicos de Investigación:

1. Las invenciones realizadas por el personal investigador de los Centros y Organismos Públicos de Investigación de la Administración General del Estado, de los Centros y Organismos de investigación de otras Administraciones Públicas, de las Universidades Públicas, de las Fundaciones del Sector Público Estatal y de las Sociedades Mercantiles Estatales pertenecerán a las entidades cuyos investigadores las hayan obtenido en el ejercicio de las funciones que les son propias, cualquiera que sea la naturaleza de la relación jurídica por la que estén vinculados a ellas.

A estos efectos se considera en todo caso personal investigador el definido como tal en el artículo 13 de la Ley 14/2011, de 1 de junio, de la Ciencia, la Tecnología y la Innovación, el personal técnico considerado en dicha Ley como personal de investigación y el personal técnico de apoyo que, conforme a la normativa interna de las universidades y de los centros de investigación, también tenga la consideración de personal de investigación.

2. Las invenciones contempladas en el apartado 1 deberán ser comunicadas por escrito a la entidad pública a cuyo servicio se halle el investigador autor de la misma en el plazo de tres meses desde la conclusión de la invención. La falta de comunicación 
por parte del personal investigador llevará consigo la pérdida de los derechos que se le reconocen en los apartados siguientes.

3. El organismo o la entidad pública, en el plazo de tres meses contados desde la recepción de la notificación a que se refiere el apartado precedente, deberán comunicar por escrito al autor o autores de la invención su voluntad de mantener sus derechos sobre la invención, solicitando la correspondiente patente, o de considerarla como secreto industrial reservándose el derecho de utilización sobre la misma en exclusiva. No podrá publicarse el resultado de una investigación susceptible de ser patentada antes de que transcurra dicho plazo o hasta que la entidad o el autor hayan presentado la solicitud de patente.

Si el organismo o entidad pública no comunica en el plazo indicado su voluntad de mantener sus derechos sobre la invención, el autor o autores de la misma podrán presentar la solicitud de patente de acuerdo con lo previsto en el artículo 18.2.

4. El investigador tendrá en todo caso derecho a participar en los beneficios que obtengan las entidades en las que presta sus servicios de la explotación o de la cesión de sus derechos sobre dichas invenciones, cuando la patente se solicite a nombre de la entidad o se decida el secreto industrial. Estas entidades podrán también ceder la titularidad de dichas invenciones al autor de las mismas, reservándose una licencia no exclusiva, intransferible y gratuita de explotación o una participación de los beneficios que se obtengan de la explotación de esas invenciones determinada de conformidad con lo dispuesto en los apartados 6 y 7.

5. En los contratos o convenios que las entidades a que se refiere el apartado 1 celebren con entes públicos o privados, se deberá estipular a quién corresponderá la titularidad de las invenciones que el personal investigador pueda realizar en el marco de dichos contratos o convenios, así como todo lo relativo a los derechos de uso y explotación comercial y al reparto de los beneficios obtenidos.

6. El Consejo de Gobierno de la Universidad determinará las modalidades y la cuantía de la participación del personal investigador de la Universidad en los beneficios que se obtengan con la explotación de las invenciones contempladas en este artículo, y en su caso, de la participación de la Universidad en los beneficios obtenidos por el investigador con la explotación de las mismas, sin perjuicio de lo establecido en el artículo 83 de la Ley Orgánica 6/2001, de 21 de diciembre, de Universidades y en el artículo 64 de la Ley 2/2011, de 4 de marzo, de Economía Sostenible.

7. Las modalidades y cuantía de la participación del personal investigador de los Entes Públicos de Investigación en los beneficios que se obtengan de la explotación 
o cesión de las invenciones contempladas en este artículo se establecerán por el Gobierno atendiendo a las características concretas de cada Ente Público de Investigación. Esta participación no tendrá en ningún caso naturaleza retributiva o salarial. Las Comunidades Autónomas podrán desarrollar por vía reglamentaria regímenes específicos de participación en beneficios para el personal investigador de Entes Públicos de Investigación de su competencia.

1.2. Real Decreto 2245/1986, de 10 de octubre, por el que se aprueba el Reglamento para la ejecución de la Ley 11/1986, de 20 de marzo, de Patentes ${ }^{6}$, modificado por el Real Decreto 151/1996, de 2 de febrero, por el que se modifica parcialmente el Reglamento de ejecución de la Ley 11/1986, de 20 de marzo, de Patentes, aprobado por Real Decreto 2245/1986, de 10 de octubre $^{7}$, y Real Decreto 245/2010, de 5 de marzo, por el que se modifica el Real Decreto 2245/1986, de 10 de octubre, por el que se aprueba el Reglamento para la ejecución de la Ley 11/1986, de 20 de marzo, de Patentes ${ }^{8}$.

1.3. Real Decreto 55/2002, de 18 de enero, sobre explotación y cesión de invenciones realizadas en los entes públicos de investigación, de conformidad con lo establecido en el artículo 20 de la Ley 11/1986, de 20 de marzo, de Patentes 9

1.4. Ley Orgánica 6/2001, de 21 de diciembre, de Universidades ${ }^{10}$, modificada por Ley Orgánica 4/2007, de 12 de abril $^{11}$, que en su artículo 1.2, c), al referirse a las funciones de la Universidad, se refiere a: "La difusión, la valorización y la transferencia del conocimiento al servicio de la cultura, de la calidad de la vida, y del desarrollo económico”.

1.5. La Ley 14/2011, de 1 de junio, de la Ciencia, la Tecnología y la Innovación $^{12}$, que además de precisar en su artículo 1 que tiene como objeto establecer "el marco para el fomento de la investigación científica y técnica y sus instrumentos de coordinación general, con el fin de contribuir a la generación, difusión y transferencia del conocimiento para resolver los problemas esenciales de la sociedad", dedica su Título in al impulso de la investigación científica y técnica, la innovación, la transferencia del conocimiento, la difusión y la cultura científica, tecnológica e innovadora.

6. вое n. ${ }^{\circ} 261$, de 31 de octubre de 1986.

7. вое n. ${ }^{\circ} 33$, de 7 de febrero de 1996.

8. вое n. ${ }^{\circ} 71$, de 23 de marzo de 2010.

9. вов n. ${ }^{\circ} 26$, de 30 de enero de 2002.

10. вое n. ${ }^{\circ} 307$, de 24 de diciembre de 2001.

11. вое n. ${ }^{\circ} 89$, de 13 de abril de 2007.

12. воE n. ${ }^{\circ} 131$, de 2 de junio de 2011. Más ampliamente, cfr. AA.vv. Régimen jurídico de la transferencia de resultados de investigación: de la Ley Orgánica de Universidades a la Ley de la Ciencia, la Tecnología y la Innovación, coord. por Carlos Vargas Vasserot, Madrid, La Ley, Grupo Wolters Kluwer, 2012. 
El artículo 33, referente a las medidas, establece en su apartado c):

Medidas para la valorización del conocimiento, que incluirán la potenciación de la actividad de transferencia desde los agentes públicos de ejecución a través de las oficinas de transferencia de resultados de investigación, y desde los parques científicos y tecnológicos, los centros tecnológicos y otras estructuras dinamizadoras de la innovación, así como el fomento de la cooperación de los agentes públicos de ejecución con el sector privado a través de los instrumentos que establece el ordenamiento jurídico y, en particular, mediante la participación en sociedades mercantiles en los términos previstos en la Ley 2/2011, de 4 de marzo, de Economía Sostenible, con el objeto de favorecer la diversificación empresarial y la transformación de los resultados de la investigación científica y técnica en desarrollo económico y social sostenible. También se impulsarán medidas de transferencia del conocimiento no orientadas a la comercialización o a la explotación mercantilizada, como la creación de espacios públicos comunes.

1.6. Ley $2 / 2011$, de 4 de marzo, de Economía Sostenible ${ }^{13}$, que establece como uno de los objetivos en materia universitaria, en el artículo 60, g): "Impulsar medidas de atracción de capital privado nacional e internacional para contribuir a la financiación de los objetivos de la universidad, especialmente a la investigación, transferencia del conocimiento y a la creación de empresas innovadoras de base tecnológica".

Por su parte, el artículo 64, sobre la investigación y transferencia del conocimiento, dispone:

1. Las universidades potenciarán sus funciones de investigación básica y aplicada y de transferencia del conocimiento a la sociedad para la mejora del bienestar y la competitividad, mediante el desarrollo de proyectos e iniciativas en colaboración con el sector productivo.

2. La colaboración entre las universidades y el sector productivo podrá articularse mediante cualquier instrumento admitido por el ordenamiento jurídico y, en particular, podrá adoptar las siguientes modalidades:

a) La constitución de empresas innovadoras de base tecnológica.

b) La generación de polos de innovación, mediante la concurrencia en un mismo espacio físico de centros universitarios y de empresas. 
c) La puesta en marcha y la potenciación de programas de valorización y transferencia de conocimiento.

d) La formación de consorcios de investigación y transferencia del conocimiento.

e) La creación de cátedras-empresa basadas en la colaboración en proyectos de investigación que permitan a los estudiantes universitarios participar y conciliar su actividad investigadora con la mejora de su formación.

3. Las universidades podrán promover la creación de empresas innovadoras de base tecnológica, abiertas a la participación en su capital societario de uno o varios de sus investigadores, al objeto de realizar la explotación económica de resultados de investigación y desarrollo obtenidos por éstos. Dichas empresas deberán reunir las características previstas en el artículo 56 de esta Ley.

En cuanto a esta legislación, nos interesa especialmente lo indicado en el Real Decreto 55/2002, ya que establece las pautas sobre la explotación y cesión dentro del marco del artículo 20 de la Ley de Patentes y el artículo 21 de la nueva Ley de Patentes, de 2015, que estará vigente el 1 de abril de 2017.

Los principales aspectos que trata esta norma son los siguientes:

- La titularidad de las invenciones, que corresponde a los organismos públicos de investigación.

- La obligación de notificación, por escrito, de la invención por parte del personal investigador.

- La evaluación por parte del organismo y comunicación al autor de la decisión sobre la invención.

- La prohibición de publicar un resultado de una investigación susceptible de ser patentada durante el plazo de 3 meses o hasta la presentación de la solicitud de patente.

- Si el organismo público de investigación decide no mantener los derechos sobre la invención, el autor podrá presentarla, teniendo el organismo un derecho a una licencia no exclusiva, intransferible y gratuita de explotación. Cuando se obtengan beneficios de la explotación corresponderá al organismo público de investigación una participación del 20\%.

- La distribución de los beneficios que obtenga un organismo público de investigación por la explotación de una invención serán de 1/3 para el mismo y $1 / 3$ para el (los) autor(es), y un 1/3 que se distribuirá de acuerdo con los criterios del Consejo Rector del organismo.

- La invención derivada de contratos o convenios de los organismos públicos de investigación, con un Ente público o privado, seguirán las especificaciones 
que indique el contrato al respecto. Los beneficios que pueda percibir el indicado organismo público se distribuirán según lo preceptuado anteriormente.

\section{La normativa de la Universitat Politècnica de València PARA LA PROPIEDAD INDUSTRIAL}

Respecto a la normativa aplicable a las patentes e invenciones en el seno de la UPV hay que indicar la siguiente:

1. El artículo 137 del Decreto 182/2011, de 25 de noviembre, del Consell, por el que se aprueban los Estatutos de la UPv ${ }^{14}$, establece en cuanto a los resultados de la investigación:

1. La Universitat gestionará las patentes e invenciones realizadas por todo su personal como consecuencia de su actividad, de acuerdo con la legislación aplicable en materia de propiedad intelectual e industrial.

2. El Consejo de Gobierno regulará las cuestiones relativas al uso de los resultados de la investigación y la atribución de los rendimientos económicos que puedan derivarse, así como la distribución del beneficio obtenido por la explotación y cesión de las patentes, entre la propia Universitat, la unidad en la que se ha realizado la investigación y las personas que han llevado a cabo la investigación.

3. Los contratos que celebre la Universitat con personas, universidades o entidades públicas y privadas para la realización de trabajos de carácter científico, técnico o artístico, así como para el desarrollo de enseñanzas de especialización o actividades específicas de formación, se realizarán conforme a la normativa que desarrolle el Consejo de Gobierno y de acuerdo con la legislación que lo regule.

4. El Consejo de Gobierno regulará la normativa de creación, reconocimiento y relación de las spin-off creadas a partir de patentes, resultados generados por proyectos de investigación o de transferencia de conocimiento en la Universitat, así como la gestión de la cartera de participaciones en las mismas. En todo caso, la creación o participación será acordada por el Consejo Social a propuesta del Consejo de Gobierno.

2. Reglamento regulador de la gestión de las actividades de investigación, desarrollo, transferencia de tecnología y formación permanente en la UPV, aprobado 
por el Consejo de Gobierno de 16 de diciembre de 2010 ${ }^{[15]}$, modificado parcial por Consejo de Gobierno de 3 de noviembre de $2011^{[16]}$.

3. Reglamento de protección y transferencia de derechos de propiedad intelectual e industrial de la upv, aprobado por Consejo de Gobierno de 9 de noviembre de $2012^{[17]}$, que deroga la anterior normativa de la propiedad industrial e intelectual aprobada por Junta de Gobierno el 21 de diciembre de 1999.

4. Código de gestión de conflictos de intereses de investigación-upv, aprobado por Consejo de Gobierno de 9 de noviembre de $2012^{[18]}$.

5. Política de integridad científica y buenas prácticas en investigación-upv, aprobado por Consejo de Gobierno de 9 de noviembre de 2012 ${ }^{[19]}$.

De toda la normativa referenciada podemos extraer los siguientes puntos a tener en cuenta:

- La normativa específica de la upv se centra en la titularidad de los resultados de las actividades de investigación, desarrollo e innovación, en la comunicación de los resultados de investigación, desarrollo e innovación susceptibles de protección jurídica y transferencia, en la protección mediante patentes y modelos de utilidad, en la promoción y comercialización de patentes, en la distribución de los ingresos provenientes de licencias o cesiones de patentes, en otras formas de protección de la propiedad industrial, en know how, así como en la propiedad intelectual e industrial derivada de contratos de I+D y en los acuerdos de confidencialidad y de transferencia de material.

- El ámbito de aplicación radica en los resultados que se generen como consecuencia de las actividades de $\mathrm{I}+\mathrm{D}+\mathrm{i}$ realizadas por el personal de la UPV en el desempeño de sus funciones, y con independencia de la relación jurídica vinculante con la misma.

- Le corresponden a la upv los derechos de propiedad industrial que se generen por los resultados derivados de subvenciones de terceros o de financiación interna. En los casos de los derechos derivados de contratos con empresas, se atenderá a lo indicado en el contrato para determinar la titularidad de los resultados.

15. Disponible en: http://www.upv.es/entidades/vi/menu_urlc.html?/entidades/vi/infoweb/ vi/info/U0534778.pdf Consultada el 24 de febrero de 2015.

16. Disponible en: http://www.upv.es/entidades/vi/menu_urlc.html?/entidades/vi/infoweb/ vi/info/U0565513.pdf Consultada el 24 de febrero de 2015.

17. Disponible en: http://www.upv.es/entidades/стт/info/U0587084.pdf Consultada el 24 de febrero de 2015). Anteriormente estaba vigente el Acuerdo de la Junta de Gobierno de 21 de diciembre de 1999: http://www.upv.es/entidades/vi/infoweb/vi/info/216007normalc. html Consultada el 24 de febrero de 2015.

18. Disponible en: http://www.upv.es/entidades/vi/menu_urlc.html?/entidades/vi/infoweb/ vi/info/U0586865.pdf Consultada el 24 de febrero de 2015.

19. Disponible en: http://www.upv.es/entidades/vi/menu_urlc.html?/entidades/vi/infoweb/ vi/info/U0586866.pdf Consultada el 24 de febrero de 2015. 
- En el caso de resultados que se obtengan por parte de personal no vinculado laboralmente, funcionarial o beca se regularán en el acuerdo de cotitularidad o cesión.

- Se reconoce el derecho moral al inventor que ostenta la persona que haya contribuido intelectualmente a la obtención de los resultados, debiendo colaborar en los procesos de protección y explotación de los mismos.

- Se establece el deber de comunicación por parte del personal de la UPV que realice una actividad de investigación, desarrollo o innovación, cuando haya resultados susceptibles de explotación económica, de comunicarlos, por escrito, al Centro de Apoyo a la Innovación, la Investigación y la Transferencia de Tecnología (en adelante стт).

- Se privilegia la protección de la invención mediante patente frente al modelo de utilidad.

- Se establece la participación de los inventores de las patentes en los beneficios de la explotación.

- Se contemplan otras formas de protección de la propiedad industrial como los diseños, según lo establecido en la Ley 20/2003, de 7 de julio, de protección jurídica del diseño industrial2 ${ }^{20}$, y en el Real Decreto 1937/2004, de 27 de septiembre, por el que se aprueba el Reglamento de ejecución de la Ley 20/2003, de 7 de julio, de protección jurídica del diseño industrial ${ }^{21}$.

- Las marcas y signos distintivos vinculados a una actividad de I+D+i de la UPV y que fueran susceptibles de ser registradas ante una Oficina de Marcas, se comunicarán al igual que las innovaciones ${ }^{22}$. El CTT será el organismo encargado para la tramitación del título y la negociación de las licencias.

- Se contempla también que en el proceso de análisis de la protección del resultado se valore si se requiere el mantenimiento del secreto, y se podrá proceder con el licenciatario un contrato de know-how ${ }^{23}$.

- Se dispone la posibilidad de establecer acuerdos con entidades terceras en cuanto a la propiedad industrial derivados de contratos de $\mathrm{I}+\mathrm{D}$, siguiendo lo indicado en la Ley Orgánica 6/2001, de Universidades.

20. воE n.o 162 , de 8 de julio de 2003.

21. вое n. ${ }^{\circ} 250$, de 16 de octubre de 2004

22. Así lo establece, refiriéndose expresamente a las innovaciones, el artículo 50 del Reglamento de protección y transferencia de derechos de propiedad intelectual e industrial de la Universitat Politècnica de València, aprobado por el Consejo de Gobierno, de 9 de noviembre de 2012. Disponible en: https://www.upv.es/entidades/SG/infoweb/sg/info/ U0587682.pdf Consultado el 29 de septiembre de 2015.

23. Sobre ello, cfr. Miguel Asensio, P. A. DE. Contratos internacionales sobre derecho de propiedad y "know-how" (conocimientos técnicos secretos), Madrid, Universidad Complutense, 2001. 
- La creación de spin-off para la transferencia de tecnología. Hasta el momento se han creado diversas empresas, participadas algunas por la UPv, a las que se les identifica con la marca ${ }^{24}$.

- Se regulan los acuerdos de confidencialidad y transferencia de material sujetos a derechos de propiedad industrial que podrán ser suscritos por el PDI de la plantilla de la upv.

\section{Algunas dudas habituales Que se plantean \\ EN LAS INGENIERÍAS RESPECTO A LA PROPIEDAD INDUSTRIAL \\ EN EL SENO DE LA UNIVERSIDAD}

En la UPV se articula la innovación, investigación y transferencia de tecnología a través del centro de apoyo denominado CTT $^{25}$. Esta unidad se encarga de la gestión de las actividades de generación de conocimiento dentro de la upv. También se encarga de la gestión de la justificación de las ayudas, internas y externas, de los investigadores, así como de la conexión con el mundo empresarial y, en general, de la canalización de todas las actividades de $\mathrm{I}+\mathrm{D}+\mathrm{I}$ del personal de la upv.

Hay que tener en cuenta la estructura en Departamentos, Institutos universita$\operatorname{rios}^{26}$ (CMT-Motores Térmicos; Aplicaciones de las Tecnologías de la Información; Automática e Informática Industrial; Conservación y Mejora de la Agrodiversidad Valenciana; Ingeniería de Alimentos para el Desarrollo; Ingeniería del Agua y del Medio Ambiente; Matemática Multidisciplinar; Matemática Pura y Aplicada; Restauración del Patrimonio; Tecnología Nanofotónica; Telecomunicación y Aplicaciones Multimedia), y los Institutos universitarios mixtos y concertados (Biología Molecular y Celular de Plantas; Biomecánica de Valencia; Tecnología de Informática; Tecnología Química), así como las estructuras propias de investigación (Centros de Investigación; Centros de Investigación mixtos y concertados; Centros de Investigación interuniversitarios, y Centros en red) y grupos de $\mathrm{I}+\mathrm{D}+\mathrm{i}^{27}$, y que en el seno de ellas se producen resultados que es preciso proteger.

El investigador, sea Licenciado, Ingeniero o actualmente Graduado, así como los becarios, suele formularse algunas cuestiones en torno a esa protección del

24. Cfr. las diversas empresas spin-off en: http://www.upv.es/entidades/CTT/ info/797751normalc.html Consultada el 25 de febrero de 2015. Una de las últimas es la empresa Diseño de alimentos S.L. que tiene por finalidad proveer de servicios de diseño de alimentos de alta calidad a empresas Food Service. Cfr. la noticia en: http://www.upv. es/entidades/Стт/noticia_846379c.html Consultada el 25 de febrero de 2015.

25. Cuya página web es: www.ctt.upv.es Consultada el 24 de febrero de 2015.

26. Se puede acceder a través de: http://www.upv.es/investigacion/estructuras/institutosuniversitarios-es.html Consultada el 24 de febrero de 2015.

27. Se pueden consultar en: https://www.upv.es/investigacion/estructuras/index-es.html Consultada el 25 de febrero de 2015. 
resultado obtenido. Es verdad que en muchas ocasiones carece de la formación específica deseable en materia de propiedad industrial, ya que los planes de estudios pasados y actuales no suelen dedicar demasiada atención a esta disciplina ${ }^{28}$.

Una de las dudas que se suelen plantear es la de qué engloba la propiedad industrial, y no se sabe diferenciarla de la propiedad intelectual. Además de tener una legislación diferente, en el ámbito de la propiedad industrial quedan incluidos la patente, los modelos de utilidad, las marcas y el diseño.

También suelen despertar inquietud los requisitos ${ }^{29}$ que debe reunir la patente, especialmente el de la novedad, ya que en muchas ocasiones se plantea si determinada actividad va a influir en dicha novedad (presentación de resultados en publicaciones), con lo que se hace preciso indicar lo permitido y lo que no lo es. Menos dudas suelen producir los requisitos de actividad inventiva y la aplicación industrial. Teniendo en cuenta, además, que todos estos requisitos son conjuntos y no excluyentes.

Las cuestiones relativas a si se deben comunicar los resultados a la Universidad, así como el porcentaje de participación, también suelen ser objeto de consulta. En muchas ocasiones, el investigador considera que tiene "propiedad sobre la patente", o, en otros términos, se dice: "Sí es mía, porque lo he hecho yo", y ello no es exactamente así. En el ámbito universitario, como hemos visto en la normativa específica de la UPV, se reconoce un porcentaje de participación en los beneficios, ya que el investigador utiliza los recursos facilitados por la entidad. En el caso de la upv, el inventor tiene el $60 \%$ y la Universidad el $40 \%$ de los beneficioes, por concepto de reinversión del sistema.

La normativa indicada permite que el profesor pueda patentar en su propio nombre, si la Universidad no lo hace (es decir, cuando la entidad académica previamente ha decidido no ejercer sus derechos de propiedad sobre la invención). En este caso se tiene un derecho a una licencia ${ }^{30}$ por parte de la Universidad, de carácter gratuito, no exclusiva e intransferible, y también una participación

28. Indicar, a título de ejemplo, que se implantó durante un breve periodo de tiempo, como libre elección, la asignatura "Protección jurídica de la invención biotecnológica", dirigida principalmente a la licenciatura en biotecnología, y en la que se proporcionaban al alumno los conocimientos básicos en materia de patentes. También, actualmente, en la asignatura Derecho Espacial, de libre elección, se le facilitan al alumno, aunque sea brevemente, ciertos conocimientos sobre la protección de los resultados que se puedan obtener en la Estación Espacial, así como ejemplos de patentes de motores, para cubrir dicha necesidad básica.

29. Díaz Muñoz, M. "Patentabilidad: concepto y requisitos", Serie Bibliotecología y Gestión de Información, n. ${ }^{\circ}$ 31, 2007, pp. 1-22.

30. Más ampliamente se puede ver esta figura en: https://www.upv.es/entidades/CTt/ info/U0670344.pdfl Consultada el 29 de septiembre de 2015. Sobre las licencias, cfr.: http://www.upv.es/entidades/Стт/conocimiento/901986normalc.html Consultado el $29 \mathrm{de}$ septiembre de 2015. 
económica del $20 \%$ en los beneficios derivados de la explotación que realice el profesor $^{31}$.

El proceso para la protección de los resultados en la upv tiene unas fases que son las siguientes ${ }^{32}$ :

1. Comunicación al CTT de los resultados, con la descripción de los mismos, personas y origen de la actividad.

2. Análisis de la patentabilidad y del potencial de la explotación por parte del Centro, a través de un informe que se dirige a la Comisión de $\mathrm{I}+\mathrm{D}+\mathrm{i}$ de la upv.

3. Registro del resultado en el Catálogo de Oferta Tecnológica de la Upv $(\text { CARTA })^{33}$. Se trata de un catálogo en el que se contiene el conocimiento que es transferible a las empresas, bien mediante patente, software o de capacidades de investigación y desarrollo. Se encuentra disponible en la página web: www.upv. es/carta y tiene estructurada la búsqueda por áreas de conocimiento, sector económico y unidades de investigación.

4. Decisión por parte de la Comisión de la solicitud de la patente.

5. Si se decide patentar, se gestiona por parte del стт la redacción y solicitud con el inventor y, en su caso, un Agente de la Propiedad Industrial (API).

6. En los casos de extensión internacional de la patente se analizan por parte del стт las acciones de comercialización, se elabora un informe y la Comisión de $\mathrm{I}+\mathrm{D}+\mathrm{i}$ decide sobre la misma.

Con la finalidad de despejar algunas dudas, dentro del Programa docencia en red, y con destino a toda persona interesada, se grabaron distintos objetos de aprendizaje $^{34}$ relacionados con las propiedad industrial, de libre acceso y conteniendo los conceptos básicos:

1. Tipos de documentos de patentes ${ }^{35}$.

31. Sobre ello, cfr.: http://www.upv.es/entidades/CTт/propiedad-intelectual/890620normalc. html Consultada el 26 de febrero de 2015.

32. Expuestas en: http://www.upv.es/entidades/стт/propiedad-intelectual/890620normalc. html Consultada el 26 de febrero de 2015.

33. Carta. Disponible en: http://www.upv.es/entidades/CtT/info/U0669180.pdf Consultado el 29 de septiembre de 2015, y también en: http://www.upv.es/carta/ Consultado el 29 de septiembre de 2015.

34. Ramón Fernández, F. "La utilización de los objetos de aprendizaje como nueva tecnología aplicada a la enseñanza técnica de la biotecnología. Su aplicación en la asignatura de Protección Jurídica de la Invención Biotecnológica", Jornada de Innovación Docente en la Escuela Técnica Superior de Ingenieros Agrónomos, Valencia, 2009, pp. 181-185.

35. Véase: https://polimedia.upv.es/polimedia.v2/lanza/index.asp?id=2af83274-aba8-8842a997-55277adfb302\&existe $400=$ Consultada el 25 de febrero de 2015. 
2. Clasificación internacional de patentes ${ }^{36}$.

3. ¿Qué es la propiedad industrial? ${ }^{37}$.

4. La patente ${ }^{38}$.

5. Distinción entre lo patentable y lo no patentable ${ }^{39}$.

\section{Conclusiones}

La protección de los resultados de la propiedad industrial en el seno de una Universidad plantea, en ocasiones, dudas para el inventor. Tenemos que tener en cuenta que existe una legislación general y una normativa específica que es aplicable, y que es propia de la Universidad a la que es aplicable.

Muchas veces el desconocimiento de cuáles son los pasos a seguir por parte del inventor hace que sea necesario que se proporcionen los conocimientos básicos en materia de propiedad industrial durante el estudio del mapa de titulaciones.

En el caso de la UPV, según indica el Reglamento de protección y transferencia de derechos de propiedad intelectual e industrial, el Vicerrectorado promoverá la formación en propiedad industrial en los estudios de ingeniería de grado y posgrado, así como la Escuela de Doctorado garantizará la formación básica de los doctorandos en la Universitat en la protección de la propiedad industrial y en la transferencia de tecnología. También se indica que los servicios de formación del Personal de Administración y Servicios (PAS) y Personal Docente e Investigador (PDI) promoverán la formación en propiedad industrial, apoyando el СТT en las acciones formativas.

La normativa específica de la UPV resuelve algunas de las dudas más habituales y establece con claridad la hoja de ruta para la protección de los resultados.

\section{Bibliografía}

AA.VV. Régimen jurídico de la transferencia de resultados de investigación: De la Ley Orgánica de Universidades a la Ley de la Ciencia, la Tecnología y la Innovación, coord. por Carlos Vargas Vasserot, Madrid, La Ley, Grupo Wolters Kluwer, 2012.

36. Véase: https://polimedia.upv.es/polimedia.v2/lanza/index.asp?id=cb2f757f-1d48-744b8a2f-263c981d33be\&existe400= Consultada el 25 de febrero de 2015.

37. Véase: https://polimedia.upv.es/polimedia.v2/lanza/index.asp?id=328d6c07-8ff5-0a4e8ed5-8453870fd2da\&existe400= Consultada el 25 de febrero de 2015.

38. Véase: https://polimedia.upv.es/polimedia.v2/lanza/index.asp?id=ae98078c-fd89-1d4f8450-350b9b11a005\&existe400= Consultada el 25 de febrero de 2015.

39. Véase: https://polimedia.upv.es/polimedia.v2/lanza/index.asp?id=cc69202b-d9976b47-a5ac-76f09805a7108existe400= Consultada el 25 de febrero de 2015. 
Caballero Fernández, G.; Quintás Corredoira, Ma. de los A., Arévalo Tomé, R. y Piñeiro García, Ma. P. "La protección de las invenciones mediante patentes en las universidades europeas, japonesas y estadounidenses”, Cuadernos de Gestión, vol. 12 (1), 2012, pp. 15-38.

Canós Darós, L., Ramón Fernández, F. y Mauri Castelló, J. J. “Aspectos jurídicos y económicos de la propiedad industrial de la empresa", Novática, n. ${ }^{\circ}$ 193, mayo-junio 2008, pp. 56-58.

Díaz Muñoz, M. "Patentabilidad: concepto y requisitos", Serie Bibliotecología y Gestión de Información, n. ${ }^{\circ}$ 31, 2007, pp. 1-22.

Miguel Asensio, P. A. DE. Contratos internacionales sobre derecho de propiedad y "know-how" (conocimientos técnicos secretos), Madrid, Universidad Complutense, 2001.

Ramón Fernández, F. “La utilización de los objetos de aprendizaje como nueva tecnología aplicada a la enseñanza técnica de la biotecnología. Su aplicación en la asignatura de Protección Jurídica de la Invención Biotecnológica”, Jornada de Innovación Docente en la Escuela Técnica Superior de Ingenieros Agrónomos, Valencia, 2009, pp. 181-185.

\section{Referencias eleCtrónicas}

Carta. Disponible en: http://www.upv.es/entidades/CтT/info/U0669180.pdf (Consultado el 29 de septiembre de 2015), y http://www.upv.es/carta/ (Consultado el 29 de septiembre de 2015).

Centro de Apoyo a la Innovación, la Investigación y la Transferencia de Tecnología. Disponible en: www.ctt.upv.es (Consultada el 24 de febrero de 2015).

Código de Gestión de Conflictos de Intereses en Investigación. Disponible en: http:// www.upv.es/entidades/vi/menu_urlc.html?/entidades/vi/infoweb/vi/info/ U0586865.pdf (Consultada el 24 de febrero de 2015).

Cuestiones básicas sobre protección de resultados en la UPV. Disponible en: http:// www.upv.es/entidades/CTT/info/712750normalc.html (Consultada el 26 de febrero de 2015).

Cuestiones clave en licencias en tecnología en la UPV. Disponible en: http://www. upv.es/entidades/Стт/info/712731 normalc.html (Consultada el 26 de febrero de 2015).

Empresas spin-off en la UPV. Disponible en: http://www.upv.es/entidades/CTT/ info/797751 normalc.html (Consultada el 25 de febrero de 2015).

Estatutos de la UPV. Disponible en: http://www.upv.es/organizacion/documentos/ estatutos-upv-2011.pdf (Consultado el 24 de febrero de 2015).

Estructuras e institutos universitarios en la UPV. Disponible en: http://www.upv. es/investigacion/estructuras/institutos-universitarios-es.html (Consultada el 24 de febrero de 2015). 
Disponible en: https://www.upv.es/investigacion/estructuras/index-es.html (Consultada el 25 de febrero de 2015).

Grados de la UPV. Disponible en: http://www.upv.es/estudios/grado/index-es. html (Consultada el 24 de febrero de 2013).

Licencias. Disponible en: http://www.upv.es/entidades/ctт/ conocimiento/901986normalc.html (Consultado el 29 de septiembre de 2015:

Normas a aplicar en las actividades de investigación, desarrollo, transferencia de tecnología y formación permanente. Disponible en: http://www.upv.es/entidades/VI/ infoweb/vi/info/216007normalc.html (Consultada el 24 de febrero de 2015).

Normativa sobre la creación de empresas en la Universidad Politécnica de Valencia a partir de la actividad de investigación universitaria. Disponible en: http:// www.ctt.upv.es/docs/NormativaAprobadaConsejoGobierno_05_2008.pdf (Consultado el 29 de septiembre de 2015).

Politica de integridad científica y buenas prácticas en investigación-UPV. Disponible en: http://www.upv.es/entidades/VI/menu_urlc.html?/entidades/VI/infoweb/ vi/info/U0586866.pdf (Consultada el 24 de febrero de 2015).

Propuesta de modificación del reglamento regulador de la gestión de las actividades de investigación, desarrollo, transferencia de tecnología y formación permanente en la UPV. Disponible en: http://www.upv.es/entidades/vi/menu_urlc.html?/ entidades/VI/infoweb/vi/info/U0565513.pdf (Consultada el 24 de febrero de 2015).

Reglamento de protección y transferencia de derechos de propiedad intelectual e industrial de la UPV. Disponible en: http://www.upv.es/entidades/ctr/info/ U0587084.pdf (Consultada el 24 de febrero de 2015), y en https://www. upv.es/entidades/SG/infoweb/sg/info/U0587682.pdf (Consultado el 29 de septiembre de 2015).

Reglamento regulador de la gestión de las actividades de investigación, desarrollo, transferencia de tecnología y formación permanente en la UPV. Disponible en: http://www.upv.es/entidades/vi/menu_urlc.html?/entidades/VI/infoweb/vi/ info/U0534778.pdf (Consultada el 24 de febrero de 2015).

\section{Objetos de aprendizaje. Polimedia grabaciones}

Tipos de documentos de patentes. Disponible en: https://polimedia.upv.es/polimedia. v2/lanza/index.asp?id=2af83274-aba8-8842-a997-55277adfb302\&existe400= (Consultada el 25 de febrero de 2015).

Clasificación internacional de patentes. Disponible en: https://polimedia. upv.es/polimedia.v2/lanza/index.asp?id=cb2f757f-1d48-744b-8a2f263c981d33be\&existe400 = (Consultada el 25 de febrero de 2015). 
¿Qué es la propiedad industrial? Disponible en: https://polimedia.upv.es/polimedia. v2/lanza/index.asp?id=328d6c07-8ff5-0a4e-8ed5-8453870fd2da\&existe400= (Consultada el 25 de febrero de 2015).

La patente. Disponible en: https://polimedia.upv.es/polimedia.v2/lanza/index. asp?id=ae98078c-fd89-1d4f-8450-350b9b11a005\&existe400 = (Consultada el 25 de febrero de 2015).

Distinción entre lo patentable y lo no patentable. Disponible en: https://polimedia.upv.es/polimedia.v2/lanza/index.asp?id=cc69202b-d997-6b47-a5ac76f09805a710\&existe400= (Consultada el 25 de febrero de 2015). 